\title{
Impacto da Obesidade nos Resultados Hospitalares da Intervenção Coronária Percutânea: Resultados do Registro do Hospital Bandeirantes
}

\author{
Leonardo dos Santos Coelho ${ }^{1}$, Marcelo José de Carvalho Cantarelli ${ }^{1}$, Hélio José Castello Jr. ${ }^{1}$, \\ Silvio Gioppato ${ }^{1}$, Rosaly Gonçalves ${ }^{1}$, João Batista de Freitas Guimarães ${ }^{1}$, \\ Evandro Karlo Pracchia Ribeiro ${ }^{1}$, Patrícia Teixeira da Silva ${ }^{1}$, Roberto Simões de Almeida ${ }^{1}$, \\ Júlio Cesar Francisco Vardi ${ }^{1}$, Rodrigo Barreto' ${ }^{1}$, Ricardo de Gasperi
}

\begin{abstract}
RESUMO
Introdução: Pacientes obesos podem apresentar melhor evolução pós-intervenção coronária percutânea (ICP) quando comparados àqueles com índice de massa corporal (IMC) normal, o chamado "paradoxo da obesidade". Este estudo teve por objetivo verificar se esse paradoxo ocorre em nosso meio. Métodos: Incluímos neste estudo 4.957 pacientes submetidos consecutivamente a ICP. Os pacientes foram classificados em não-obesos (IMC $\left.<30 \mathrm{~kg} / \mathrm{m}^{2}\right)$ e obesos $\left(I M C \geq 30 \mathrm{~kg} / \mathrm{m}^{2}\right)$. Eventos cardíacos e cerebrovasculares adversos maiores (ECCAM) foram registrados na alta hospitalar. Resultados: O grupo de obesos apresentou-se três anos mais jovem, com maior prevalência de fatores de risco para doença arterial coronária, à exceção do tabagismo. A apresentação clínica foi semelhante, prevalecendo os quadros clínicos estáveis. Predominaram os pacientes com acometimento uniarterial, as características de complexidade das lesões não diferiram entre os grupos, à exceção de lesões calcificadas, e a disfunção ventricular esquerda foi menos frequente no grupo de obesos. O diâmetro e a extensão dos stents utilizados foram semelhantes entre os grupos. A taxa de sucesso do procedimento foi alta e similar para obesos e não-obesos. Na alta hospitalar, a incidência de $\operatorname{ECCAM}(2,5 \%$ vs. $2,7 \% ; P=0,76)$, óbito hospitalar ( $1 \%$ vs. $1,1 \% ; \mathrm{P}=0,88)$, acidente vascular cerebral $(0,1 \%$ vs. $0,1 \% ; P=0,79)$, infarto agudo do miocárdio $(1,6 \%$ vs. $1,8 \% ; P=0,74)$ e revascularização miocárdica de emergência ( 0 vs. $0,1 \%$; $P=0,35$ ) não mostrou diferenças entre os grupos. Idade, diabetes, hipertensão e lesões tipo B2/C foram as variáveis que melhor explicaram a presença de ECCAM. Conclusões: Em pacientes portadores de doença arterial coronária e submetidos a ICP, o $I M C \geq 30 \mathrm{~kg} / \mathrm{m}^{2}$ não influenciou o risco de eventos clínicos hospitalares relacionados ao procedimento.
\end{abstract}

DESCRITORES: Obesidade. Angioplastia. Stents.

\section{ABSTRACT}

Impact of Obesity on In-Hospital Outcomes of Percutaneous Coronary Intervention: Results of Hospital Bandeirantes Registry

Background: Obese patients may have better outcomes after percutaneous coronary intervention $(\mathrm{PCl})$ compared to those with normal body mass index (BMI), the so-called "obesity paradox". This study was aimed at evaluating whether this paradox is observed in our country. Methods: This study included 4,957 consecutive patients submitted to PCI. Patients were classified as non-obese $\left(\mathrm{BMI}<30 \mathrm{~kg} / \mathrm{m}^{2}\right)$ and obese $\left(\mathrm{BMI}>30 \mathrm{~kg} / \mathrm{m}^{2}\right)$. Major adverse cardiac and cerebrovascular events (MACCE) were recorded at hospital discharge. Results: The obese group was three years younger, with a higher prevalence of risk factors for coronary artery disease, except for smoking. Clinical presentation was similar, with a predominance of stable coronary patients. Single vessel disease was the most frequent finding and the complexity of the lesions was not different between groups, except for calcified lesions, and left ventricular dysfunction was less frequent in the obese group. Stent diameter and length were similar between groups. Procedure success rate was high and similar for obese and non-obese patients. At hospital discharge, the incidence of MACCE (2.5\% vs. $2.7 \%$; $\mathrm{P}=0.76)$, in-hospital death $(1 \%$ vs. $1.1 \% ; \mathrm{P}=0.88)$, stroke $(0.1 \%$ vs. $0.1 \%$; $\mathrm{P}=0.79)$, acute myocardial infarction $(1.6 \%$ vs. $1.8 \% ; P=0.74)$ and emergency CABG (0 vs. $0.1 \%$; $\mathrm{P}=0.35)$ was not different between groups. Age, diabetes, hypertension and type B2/C lesions were the variables that best explained MACCE. Conclusions: In patients with cOronary artery disease undergoing $\mathrm{PCl}, \mathrm{BMI}>30 \mathrm{~kg} / \mathrm{m}^{2}$ did not influence the risk of procedure-related in-hospital clinical events.

KEY-WORDS: Obesity. Angioplasty. Stents.
1 Hospital Bandeirantes - São Paulo, SP, Brasil.

Correspondência: Leonardo dos Santos Coelho. Rua José Getúlio, 206/33 - Liberdade - São Paulo, SP, Brasil - CEP 01509-000 E-mail: leostcoelho@gmail.com

Recebido em: 4/9/2011 • Aceito em: 20/11/2011 
A obesidade, definida como índice de massa corporal $(I M C) \geq 30 \mathrm{~kg} / \mathrm{m}^{2}$, tem crescido de forma epidêmica na sociedade moderna nas últimas décadas e contribui para o aparecimento da doença cardiovascular, por estar envolvida em processos metabólicos relacionados a hipertensão arterial, diabetes melito e dislipidemia, reconhecidos fatores de risco para a aterogênese. ${ }^{1-4}$

No Brasil, a prevalência de obesidade é de cerca de $8 \%$ para os homens e de $12,4 \%$ para as mulheres. Ao se somar pacientes com sobrepeso $\left(\mathrm{IMC} \geq 25 \mathrm{~kg} / \mathrm{m}^{2}\right)$, esses números elevam-se para 38,5\% e 39\%, respectivamente. $^{2}$ Nos Estados Unidos, mais de $50 \%$ da população está acima do peso, sendo $20 \%$ classificada como obesa pelos critérios do National Institutes of Health (NIH). ${ }^{4}$

A associação de obesidade com fatores de risco como hipertensão arterial e diabetes melito, resistência à insulina e marcadores inflamatórios elevados pode contribuir para o aumento de desfechos desfavoráveis relacionados à intervenção coronária percutânea (ICP) em obesos. ${ }^{5,6}$ No entanto, vários estudos têm sugerido um "paradoxo da obesidade", no qual a obesidade parece ser protetora contra um prognóstico adverso, marcadamente com redução da mortalidade intra-hospitalar e a longo prazo. ${ }^{7-9}$

O objetivo deste estudo foi avaliar qual o impacto atual da obesidade (IMC $\geq 30 \mathrm{~kg} / \mathrm{m}^{2}$ ) na evolução hospitalar pós-ICP.

\section{MÉTODOS}

\section{Pacientes}

No período de agosto de 2006 a novembro de 2010, 4.957 pacientes foram submetidos consecutivamente a ICP e incluídos no Registro do Hospital Bandeirantes. Os dados foram coletados de forma prospectiva e armazenados em um banco de dados informatizado.

O IMC foi calculado dividindo-se o peso em quilogramas pelo quadrado da altura em metros. Os pacientes foram classificados em não-obesos (IMC $<30 \mathrm{~kg} / \mathrm{m}^{2}$ ) e obesos (IMC $\left.\geq 30 \mathrm{~kg} / \mathrm{m}^{2}\right)$, de acordo com a classificação do $\mathrm{NIH} .{ }^{4}$ Peso e altura foram obtidos na época da ICP. As complicações hospitalares foram registradas no momento da alta hospitalar.

\section{Intervenção coronária percutânea}

As intervenções foram realizadas em quase sua totalidade por via femoral, sendo utilizada a via radial como opção em poucos casos. A técnica e a escolha do material durante o procedimento ficaram a cargo dos operadores, assim como a necessidade do uso de inibidores da glicoproteína Ilb/Illa. Foi utilizada heparina não-fracionada no início do procedimento na dose de
$70 \mathrm{U} / \mathrm{kg}$ a $100 \mathrm{U} / \mathrm{kg}$, exceto nos pacientes que já estavam em uso de heparina de baixo peso molecular. Todos os pacientes receberam terapia antiplaquetária combinada com ácido acetilsalicílico (AAS), nas doses de ataque de $300 \mathrm{mg}$ e de manutenção de 100 mg/dia a $200 \mathrm{mg} / \mathrm{dia}$, e clopidogrel, nas doses de ataque de $300 \mathrm{mg}$ a $600 \mathrm{mg}$ e de manutenção de $75 \mathrm{mg} / \mathrm{dia}$. Os introdutores femorais foram retirados quatro horas após o início da heparinização. Os introdutores radiais foram retirados imediatamente após o término do procedimento.

\section{Análise angiográfica e definições}

As análises foram realizadas em pelo menos duas projeções ortogonais, por operadores experientes, com uso de angiografia quantitativa digital. Neste estudo foram utilizados os mesmos critérios angiográficos constantes no banco de dados da Central de Intervenções Cardiovasculares (CENIC) da Sociedade Brasileira de Hemodinâmica e Cardiologia Intervencionista. O tipo de lesão foi classificado conforme os critérios do American College of Cardiology e American Heart Association (ACC/AHA). ${ }^{10}$ Foram consideradas lesões longas aquelas com comprimento > $20 \mathrm{~mm}$. Para a determinação do fluxo coronário pré e pós-procedimento foi utilizada a classificação de TIMI. ${ }^{11}$ Sucesso do procedimento foi definido como obtenção de sucesso angiográfico (estenose residual $<30 \%$, com fluxo TIMI 3) e ausência de eventos cardíacos e cerebrovasculares adversos maiores (ECCAM), compreendendo óbito, infarto periprocedimento, acidente vascular cerebral (AVC) e cirurgia de revascularização miocárdica de emergência. ${ }^{12}$

Os óbitos por qualquer causa foram contabilizados e a mortalidade cardíaca foi definida como aquela consequente ao choque cardiogênico, insuficiência cardíaca, infarto agudo do miocárdio (IAM), ruptura cardíaca, arritmia ou morte súbita no período hospitalar. O infarto peri-ICP foi definido pelo reaparecimento de sintomas anginosos, com presença de alterações eletrocardiográficas (novo supradesnivelamento do segmento ST ou novas ondas Q) e/ou evidência angiográfica de oclusão do vaso-alvo. Foi considerada cirurgia de revascularização miocárdica de emergência aquela realizada imediatamente após a ICP.

\section{Análise estatística}

Os dados armazenados em banco de dados com base Oracle foram plotados em planilhas Excel e analisados em programa estatístico SPSS versão 15.0. As variáveis contínuas foram expressas em média \pm desvio padrão e as variáveis categóricas, em percentis. As associações entre as variáveis contínuas foram avaliadas pelo teste $t$ de Student ou pelo teste de Mann-Whitney. As associações entre as variáveis categóricas foram avaliadas pelos testes qui-quadrado ou exato de Fischer, quando apropriado. Foi adotado nível de significância de $\mathrm{P}<0,05$. Modelos de regressão logística simples e 
múltipla foram aplicados para identificar preditores de ECCAM. As variáveis incluídas foram idade, sexo, IMC $\left(<30 \mathrm{~kg} / \mathrm{m}^{2}\right.$ vs. $\left.>30 \mathrm{~kg} / \mathrm{m}^{2}\right)$, hipertensão arterial sistêmica, diabetes melito, dislipidemia, tabagismo, IAM prévio, fluxo TIMI (0/1 vs. 2/3) e complexidade da lesão pela classificação do ACC/AHA (A/B1 vs. B2/C). Foi adotado nível de significância de $\mathrm{P}<0,05$.

\section{RESULTADOS}

As características clínicas estão expostas na Tabela 1. O grupo de obesos apresentou-se três anos mais jovem (59,7 anos vs. 62,7 anos; $\mathrm{P}<0,01)$, com maior prevalência de hipertensão arterial $(82,6 \%$ vs. $72,1 \%$; $\mathrm{P}<0,01)$, diabetes $(38,6 \%$ vs. $26,8 \% ; \mathrm{P}<0,01)$ e dislipidemia $(40,9 \%$ vs. 31,5\%; $\mathrm{P}<0,01)$, e menor prevalência de tabagismo $(20,7 \%$ vs. $23,7 \%$; $P=0,04)$, comparativamente ao grupo de não-obesos. A apresentação clínica foi similar entre os grupos, predominando os quadros clínicos estáveis (assintomáticos e angina estável) em 55\% dos pacientes tratados. A quase totalidade dos pacientes (> 96\%) estava em uso de
AAS e clopidogrel pré-procedimento, não ocorrendo diferença entre os grupos. Os pacientes obesos mostraram maior necessidade do uso intra-hospitalar de insulina $(8,1 \%$ vs. $5,5 \% ; \mathrm{P}=0,01)$.

A Tabela 2 apresenta as características angiográficas, similares na maioria das comparações entre obesos e não-obesos. Predominaram os pacientes com acometimento uniarterial (55\%), e a artéria descendente anterior foi o vaso mais frequentemente abordado (42\%). As lesões B2/C foram tratadas em mais da metade dos casos $(58,8 \%$ vs. $55,8 \% ; \mathrm{P}=0,16)$ e as características de complexidade das lesões não diferiram entre os grupos, à exceção das lesões calcificadas $(4,5 \%$ vs. $5,3 \% ; P=0,01)$. Disfunção ventricular esquerda foi menos frequente no grupo de obesos (15,9\% vs. $22,2 \%$; $\mathrm{P}<0,01)$.

A quantificação angiográfica das obstruções pré e pós-procedimento, o diâmetro $(3,01 \pm 0,5 \mathrm{~mm}$ vs. $2,95 \pm 0,48 \mathrm{~mm} ; \mathrm{P}=0,08)$ e a extensão $(18,6 \pm 6,9 \mathrm{~mm}$ vs. $1 \overline{8}, 3+7 \mathrm{~mm} ; \mathrm{P}=0,4)$ dos stents utilizados não

TABELA 1

Características clínicas

\begin{tabular}{|c|c|c|c|}
\hline Característica & $\begin{array}{c}\text { Obesos } \\
(n=1.052)\end{array}$ & $\begin{array}{c}\text { Não-obesos } \\
(n=3.905)\end{array}$ & Valor de $\mathbf{P}$ \\
\hline Idade, anos & $59,7 \pm 11,6$ & $62,7 \pm 11,9$ & $<0,01$ \\
\hline Sexo masculino, n (\%) & $714(67,9)$ & $2.676(68,5)$ & 0,68 \\
\hline Hipertensão arterial, n (\%) & $869(82,6)$ & $2.816(72,1)$ & $<0,01$ \\
\hline Dislipidemia, n (\%) & $430(40,9)$ & $1.232(31,5)$ & $<0,01$ \\
\hline Diabetes melito, n (\%) & $406(38,6)$ & $1.046(26,8)$ & $<0,01$ \\
\hline Tabagismo, n (\%) & $218(20,7)$ & $926(23,7)$ & 0,04 \\
\hline IAM prévio, n (\%) & $184(17,5)$ & $709(18,2)$ & 0,62 \\
\hline ICP prévia, n (\%) & $198(18,8)$ & $640(16,4)$ & 0,28 \\
\hline RM prévia, n (\%) & $122(11,6)$ & $434(11,1)$ & 0,66 \\
\hline IRC, n (\%) & $26(2,5)$ & $109(2,8)$ & 0,57 \\
\hline Apresentação clínica, n (\%) & & & 0,32 \\
\hline Assintomático & $290(27,5)$ & $1.117(28,6)$ & \\
\hline Angina estável & $272(25,9)$ & $1.047(26,8)$ & \\
\hline SCA & $231(21,9)$ & $737(18,9)$ & \\
\hline IAM & $241(22,9)$ & $934(23.9)$ & \\
\hline ICC & $19(1,8)$ & $71(1,8)$ & \\
\hline ICP primária, n (\%) & $92(8,7)$ & $401(10,3)$ & 0,62 \\
\hline \multicolumn{4}{|l|}{ Medicação adjunta, n (\%) } \\
\hline AAS & $1.024(97,3)$ & $3.788(97)$ & 0,57 \\
\hline Clopidogrel & $1.023(97,2)$ & $3.764(96,4)$ & 0,68 \\
\hline Estatina & $377(60,1)$ & $1.356(57,1)$ & 0,17 \\
\hline Inibidores da glicoproteína IIb/IIla & $70(6,7)$ & $242(6,2)$ & 0,77 \\
\hline Insulina & $51(8,1)$ & $130(5,5)$ & 0,01 \\
\hline
\end{tabular}


diferiram entre os grupos (Tabela 3). A taxa do uso de stents foi semelhante $(90,7 \%$ vs. $89,9 \% ; P=0,79)$, tendo o grupo de obesos apresentado maior utilização de stents farmacológicos (18,4\% vs. 14,7\%; $\mathrm{P}<0,01)$. A taxa de sucesso do procedimento foi alta e similar para obesos e não-obesos $(96,5 \%$ vs. 95,9\%; $P=0,37)$.

Os desfechos hospitalares (Tabela 4) da ICP não mostraram diferenças entre os grupos quanto à inci- dência de $\operatorname{ECCAM}(2,5 \%$ vs. $2,7 \%$; $P=0,76)$ e à ocorrência de óbito hospitalar (1\% vs. $1,1 \% ; \mathrm{P}=0,88)$, AVC $(0,1 \%$ vs. $0,1 \% ; P=0,79), \operatorname{IAM}(1,6 \%$ vs. $1,8 \%$; $\mathrm{P}=0,74)$ e cirurgia de revascularização miocárdica de emergência ( 0 vs. $0,1 \%$; $P=0,35)$.

À análise multivariada, idade [odds ratio (OR) 1,02, intervalo de confiança de 95\% (IC 95\%) 1,01-1,04; $P=0,003$ ), hipertensão arterial (OR 1,87, IC 95\% $1,17-2,98 ; P=0,009)$, diabetes (OR 1,56, IC 95\%

TABELA 2

Características angiográficas

\begin{tabular}{|c|c|c|c|}
\hline Característica & $\begin{array}{c}\text { Obesos } \\
(1.052 \text { pacientes/ } \\
1.526 \text { lesões) }\end{array}$ & $\begin{array}{c}\text { Não-obesos } \\
\text { (3.905 pacientes/ } \\
5.672 \text { lesões) }\end{array}$ & Valor de $\mathbf{P}$ \\
\hline Vasos acometidos, n (\%) & & & 0,47 \\
\hline Um & $595(56,6)$ & $3.057(53,9)$ & \\
\hline Dois & $305(29)$ & $1.724(30,4)$ & \\
\hline Três & $139(13,2)$ & $783(13,8)$ & \\
\hline Lesões calcificadas, n (\%) & $68(4,5)$ & $300(5,3)$ & 0,01 \\
\hline Lesões tipo B2/C, n (\%) & $897(58,8)$ & $3.165(55,8)$ & 0,16 \\
\hline Lesões longas, n (\%) & $172(11,3)$ & $794(14)$ & 0,1 \\
\hline Bifurcações, n (\%) & $242(15,9)$ & $737(13)$ & 0,09 \\
\hline Lesões com trombos, n (\%) & $44(2,9)$ & $130(2,3)$ & 0,45 \\
\hline Disfunção ventricular esquerda, n (\%) & $167(15,9)$ & $867(22,2)$ & $<0,001$ \\
\hline Fluxo TIMI pré-ICP, n (\%) & & & 0,23 \\
\hline TIMI 0/1 & $100(6,6)$ & $318(5,6)$ & \\
\hline TIMI 2/3 & $1.425(93,4)$ & $5.354(94,4)$ & \\
\hline Vaso tratado, n (\%) & & & 0,77 \\
\hline CD & $548(35,9)$ & $1.996(35,2)$ & \\
\hline $\mathrm{DA}$ & $606(39,7)$ & $2.410(42,5)$ & \\
\hline$C x$ & $358(23,5)$ & $1.204(21,2)$ & \\
\hline TCE & $14(0,9)$ & $62(1,1)$ & \\
\hline
\end{tabular}

$\mathrm{CD}$ = artéria coronária direita; $\mathrm{Cx}=$ artéria coronária circunflexa; DA = artéria descendente anterior; ICP = intervenção coronária percutânea; TCE = tronco de coronária esquerda; TIMI = Thrombolysis in Myocardial Infarction.

TABELA 3

Características dos procedimentos

\begin{tabular}{lccc}
\hline & Obesos & Não-obesos & \\
Característica & $\begin{array}{c}\mathbf{1 . 0 5 2} \text { pacientes/ } \\
\mathbf{1 . 5 2 6} \text { lesões) }\end{array}$ & $\begin{array}{c}\mathbf{( 3 . 9 0 5} \text { pacientes/ } \\
\mathbf{5 . 6 7 2} \text { lesões) }\end{array}$ & Valor de P \\
\hline Número de stents (\%) & $1.384(90,7)$ & $5.100(89,9)$ & 0,79 \\
Stents convencionais & $1.129(81,6)$ & $4.349(85,3)$ & 0,86 \\
Stents farmacológicos & $255(18,4)$ & $751(14,7)$ & $<0,01$ \\
Diâmetro dos stents, mm & $3,01 \pm 0,5$ & $2,95 \pm 0,48$ & 0,08 \\
Comprimento dos stents, mm & $18,6 \pm 6,9$ & $18,3 \pm 7$ & 0,4 \\
Grau de estenose pré, \% & $84,9 \pm 12,7$ & $83,8 \pm 12,3$ & 0,73 \\
Grau de estenose pós, \% & $1,1 \pm 7,6$ & $1,2 \pm 8$ & 0,35 \\
Sucesso do procedimento, $\mathrm{n}(\%)$ & $1.015(96,5)$ & $3.744(95,9)$ & 0,37 \\
\hline
\end{tabular}


1,05-2,34; $\mathrm{P}=0$,029) e lesões tipo B2/C (OR 2,25, IC $95 \% 1,49-3,41 ; P<0,001)$ foram as variáveis que melhor explicaram a presença de ECCAM hospitalares (Tabela 5).

\section{DISCUSSÃO}

Neste estudo foi avaliado o impacto da obesidade na evolução hospitalar de uma grande coorte de pacientes submetidos a ICP contemporânea. Os achados demonstraram que o IMC $\geq 30 \mathrm{~kg} / \mathrm{m}^{2}$ não influenciou a taxa de eventos clínicos adversos hospitalares.

Esse achado contrasta com estudo anterior, em que Hastie et al. ${ }^{8}$ demonstraram que pacientes com IMC aumentado apresentam o "paradoxo da obesidade", segundo o qual pacientes obesos apresentam taxas de complicações intra-hospitalares e tardias menores que as observadas em não-obesos. Metanálise de ensaios clínicos de procedimentos de revascularização percutânea ou cirúrgica, realizada por Oreopoulos et al. ${ }^{9}$, revelou que o sobrepeso e a obesidade exercem efeito protetor na mortalidade após procedimento a curto e longo prazos.

O mecanismo pelo qual pacientes não-obesos apresentam maior número de complicações não está claro, mas pode estar relacionado à presença de doenças não-cardiovasculares graves em pacientes muito magros. Esse achado já foi observado em nosso meio por Lemos et al. ${ }^{13}$, que constataram que $I M C \leq 20 \mathrm{~kg} / \mathrm{m}^{2}$ é fator de risco independente para a ocorrência de óbito tanto precoce como tardio pós-ICP.

Estudos prévios destacam também como provável fator positivo o fato de pacientes obesos serem mais frequentemente encaminhados para tratamentos invasivos apropriados e receberem mais frequentemente terapia medicamentosa de acordo com as diretrizes vigentes pelo maior número de comorbidades, o que acarretaria um resultado mais favorável. ${ }^{7,8}$ Uma subanálise do registro Alberta Provincial Project for Outcome Assessment in Coronary Heart Disease (APPROACH $)^{14}$, que avaliou a relação entre IMC, tratamento e mortalidade em pacientes com doença arterial coronária estabelecida, mostrou que a obesidade é um fator que faz com que os médicos encaminhem esses pacientes para tratamento invasivo mais precocemente do que pacientes com IMC normal. Foi demonstrado, ainda, em alguns estudos, que os pacientes obesos (mas não os com obesidade grave) são mais propensos a serem revascularizados, apesar de apresentarem anatomia coronária de menor complexidade. ${ }^{14}$ Nesta análise, os obesos apresentaram-se à ICP mais jovens e a variável idade foi um preditor independente de ECCAM, e o risco desses eventos aumenta $2 \%$ a cada ano. A obe-

TABELA 4

Desfechos clínicos na fase hospitalar

\begin{tabular}{lccc}
\hline Característica & $\begin{array}{c}\text { Obesos } \\
(\mathbf{n = 1 . 0 5 2 )}\end{array}$ & $\begin{array}{c}\text { Não-obesos } \\
(\mathbf{n}=\mathbf{3 . 9 0 5})\end{array}$ & Valor de P \\
\hline ECCAM, n (\%) & $27(2,5)$ & $108(2,7)$ & 0,76 \\
Mortalidade geral, n (\%) & $11(1)$ & $43(1,1)$ & 0,88 \\
Mortalidade cardíaca, n (\%) & $7(0,7)$ & $33(0,8)$ & 0,38 \\
IAM, n (\%) & $17(1,6)$ & $69(1,8)$ & 0,74 \\
RM de emergência, n (\%) & 0 & $4(0,1)$ & 0,35 \\
AVC, n (\%) & $1(0,1)$ & $5(0,1)$ & 0,79 \\
\hline
\end{tabular}

AVC = acidente vascular cerebral; ECCAM = eventos cardíacos e cerebrovasculares adversos maiores; IAM = infarto agudo do miocárdio; RM = cirurgia de revascularização miocárdica.

TABELA 5

Preditores independentes de eventos cardíacos e cerebrovasculares adversos maiores hospitalares pós-intervenção coronária percutânea

\begin{tabular}{lccc} 
Fatores selecionados & OR & IC 95\% & Valor de P \\
\hline Idade & 1,02 & {$[1,01 ; 1,04]$} & 0,003 \\
Hipertensão arterial sistêmica & 1,87 & {$[1,17 ; 2,98]$} & 0,009 \\
Diabetes & 1,56 & {$[1,05 ; 2,34]$} & 0,029 \\
Lesão B2/C & 2,25 & {$[1,49 ; 3,41]$} & 0,0001 \\
\hline
\end{tabular}

IC 95\% = intervalo de confiança de 95\%; OR = odds ratio. 
sidade, contudo, não foi um preditor independente desses desfechos clínicos desfavoráveis. Estudos mais recentes baseados em registros e que incluíram pacientes com IMC $>40 \mathrm{~kg} / \mathrm{m}^{2}$ sugerem que esses pacientes são os que apresentam maiores taxas de mortalidade. ${ }^{15}$

\section{Limitações do estudo}

São limitações do presente estudo a análise retrospectiva dos dados, sua realização em um único centro e a ausência de seguimento tardio. A classificação dos pacientes nas categorias peso abaixo do normal $\left(<18,5 \mathrm{~kg} / \mathrm{m}^{2}\right)$, sobrepeso $\left(25 \mathrm{~kg} / \mathrm{m}^{2}\right.$ a $\left.29,9 \mathrm{~kg} / \mathrm{m}^{2}\right)$ e nos estágios I $\left(30 \mathrm{~kg} / \mathrm{m}^{2}\right.$ a $\left.34,9 \mathrm{~kg} / \mathrm{m}^{2}\right)$, II $\left(35 \mathrm{~kg} / \mathrm{m}^{2}\right.$ a $\left.39,9 \mathrm{~kg} / \mathrm{m}^{2}\right)$ e III $\left(\geq 40 \mathrm{~kg} / \mathrm{m}^{2}\right)$ da obesidade poderia fornecer informações adicionais. Por fim, a definição da obesidade pelo IMC não leva em consideração a distribuição da gordura corporal, que pode ser melhor preditor de risco cardiovascular.

\section{CONCLUSÕES}

Em pacientes portadores de doença arterial coronária submetidos a ICP, a obesidade (IMC $\left.\geq 30 \mathrm{~kg} / \mathrm{m}^{2}\right)$ não influenciou o risco de eventos clínicos hospitalares relacionados ao procedimento.

\section{CONFLITO DE INTERESSES}

Os autores declaram não haver de conflito de interesses relacionado a este manuscrito.

\section{REFERÊNCIAS}

1. Ross R. The pathogenesis of atherosclerosis - an update. $N$ Engl J Med. 1986;314(8):488-500.

2. Guimarães HP, Avezum A, Piegas LS. Obesidade abdominal e síndrome metabólica. Rev Soc Cardiol Estado de São Paulo. 2006;16(1):41-7.

3. Wilson PW, D'Agostino RB, Sullivan L, Parise $H$, Kannel WB. Overweight and obesity as determinants of cardiovascular risk: the Framingham experience. Arch Intern Med. 2002; 162(16):1867-72

4. National Institutes of Health (NIH); National Heart, Lung, and Blood Institute (NHLBI). Clinical guidelines on the identification, evaluation, and treatment of overweight and obesity in adults: the evidence report. Bethesda (MD); 1998.
5. Tarastchuk JCE, Guérios EE, Bueno RRL, Andrade PMP, Nercolini DC, Ferraz JGG, et al. Obesidade e intervenção coronariana: devemos continuar valorizando o Índice de Massa Corpórea? Arq Bras Cardiol. 2008;90(5):284-9.

6. Rana JS, Mittleman MA, Ho KK, Cutlip DE. Obesity and clinical restenosis after coronary stent placement. Am Heart J. $2005 ; 150(4): 821-6$

7. Gruberg L, Weissman NJ, Waksman R, Fuchs S, Deible R, Pinnow EE, et al. The impact of obesity on the short-term and long-term outcomes after percutaneous coronary intervention: the obesity paradox? J Am Coll Cardiol. 2002; 39(4):578-84.

8. Hastie $C E$, Padmanabhan $S$, Slack R, Pell ACH, Oldroyd KG, Flapan AD, et al. Obesity paradox in a cohort of 4880 consecutive patients undergoing percutaneous coronary intervention. Eur Heart J. 2010;31(2):222-6.

9. Oreopoulos A, Padwal R, Norris CM, Mullen JC, Pretorius $V$, Kalantar-Zadeh K. Effect of obesity on short- and longterm mortality postcoronary revascularization: a meta-analysis. Obesity. 2008;16(2):442-50.

10. Smith SC Jr, Feldman TE, Hirshfeld JW Jr, Jacobs AK, Kern MJ, King SB $3^{\text {rd }}$, et al. ACC/AHA/SCAI 2005 guideline update for percutaneous coronary intervention: a report of the American College of Cardiology/American Heart Association Task Force on Practice Guidelines (ACC/AHA/SCAI Writing Committee to Update 2001 Guidelines for Percutaneous Coronary Intervention). Circulation. 2006;113(7):e166-286.

11. TIMI Study Group. The Thrombolysis in Myocardial Infarction (TIMI) trial. Phase I findings. N Engl J Med. 1985;312(14): 932-6.

12. Mattos LA, Lemos Neto PA, Rassi A Jr, Marin-Neto JA, Sousa AGMR, Devito FS, et al. Diretrizes da Sociedade Brasileira de Cardiologia - Intervenção Coronária Percutânea e Métodos Adjuntos Diagnósticos em Cardiologia Intervencionista (II Edição - 2008). Rev Bras Cardiol Invasiva. 2008;16(Supl 2):9-88.

13. Lemos PA, Ribeiro EE, Kajita LJ, Esteves Filho A, Campos $\mathrm{CAH}$, Falcão BAA, et al. Muito baixo peso ponderal em pacientes tratados com angioplastia coronária: impacto na mortalidade precoce e tardia. Rev Bras Cardiol Invasiva. 2008; 16(4):429-33.

14. Oreopoulos A, McAlister FA, Norris CM, Kalantar-Zadeh K, Padwal R, Ezekowitz JA, et al. The relationship between body mass index, treatment, and mortality in patients with established coronary artery disease: a report from APPROACH. Eur Heart J. 2009;30(21):2584-92.

15. Minutello RM, Chou ET, Hong MK, Bergman G, Parikh M, lacovone $\mathrm{F}$, et al. Impact of body mass index on in-hospital outcomes following percutaneous coronary intervention (report from the New York State Angioplasty Registry). Am J Cardiol. 2004;93(10):1229-32. 